IMSc- $94 / 28$

TIFR/TH/94-14

hep-th/9405146

Revised October 1994

\title{
A proposal for the geometry of $W_{n}$ gravity
}

\author{
Suresh Govindarajan \\ Theoretical Physics Group \\ Tata Institute of Fundamental Research \\ Bombay 400005 INDIA \\ T. Jayaraman! \\ The Institute of Mathematical Sciences \\ C.I.T. Campus, Taramani \\ Madras 600 113, INDIA
}

\begin{abstract}
We relate the Teichmuller spaces obtained by Hitchin to the Teichmuller spaces of $W A_{n}$-gravity. The relationship of this space to $W$-gravity is obtained by identifying the flat $\operatorname{PSL}(n+1, \mathbb{R})$ connections of Hitchin to generalised vielbeins and connections. This is explicitly demonstrated for $W A_{2}=W_{3}$ gravity. We show how $W$-diffeomorphisms are obtained in this formulation. We find that particular combinations of the generalised connection play the role of projective connections. We thus obtain $W$-diffeomorphisms in a geometric fashion without invoking the presence of matter fields. This description in terms of vielbeins naturally provides the measure for the gravity sector in the Polyakov path integral for $W$-strings.
\end{abstract}

Keywords: String Theory, Geometry, Teichmuller Spaces, W-gravity, Higgs Bundles.

\footnotetext{
*E-mail: suresh@theory.tifr.res.in

${ }^{\dagger}$ E-mail: jayaram@imsc.ernet.in
} 


\section{Introduction}

One of the ways new structure arises in string theory is by extending the gauge symmetry of the world sheet beyond bosonic reparametrization invariance. The most well-known is of course supersymmetry, where there are both bosonic and fermionic degrees of freedom on the world sheet leading to local supersymmetry on the world sheet. The addition of this new symmetry plays an important role in non-critical strings allowing the extension of string theories beyond the bosonic strong-coupling barrier of $c=1$. It is interesting to examine whether there are new structures associated with bosonic and/or fermionic chiral algebras (in the CFT sense) apart from the Virasoro algebra. This has been one of the prime motivations in studying $W$-strings where the $W$-algebra is the additional chiral algebra present in the theory.

Among the outstanding problems of formulating a theory of $W$-strings are first the non-linearity of the algebra [1] and the lack of knowledge of the structure of the associated moduli and Teichmuller spaces, especially the measure. In the Polyakov path integral formulation of string theory, one can show that the integration over all metrics can be reduced to one over the moduli space of Riemann surfaces. Associated to this moduli space is the Teichmuller space which when quotiented by the mapping class group gives moduli space. Not much is known about this space in the case of $W$-strings except for the dimension, obtained by counting the number of zero modes of the associated ghost systems. The other problem is of course the insufficient understanding of $W$-coordinates which is especially difficult given the bosonic nature of the $W$-symmetry. Several attempts have been made in the literature to tackle these issues but the problems remain largely unresolved. In this paper we make an attempt to tackle the first problem, basing ourselves on the work of Hitchin on Higgs bundles [2].

In the case of the bosonic string, it is known that the Teichmuller space can be obtained as a particular component of the space (for a surface $\Sigma$ with genus 
$g>1) \mathcal{T}_{2}(\Sigma) \equiv \operatorname{Hom}\left(\pi_{1}(\Sigma) \rightarrow \operatorname{PSL}(2, \mathbb{R})\right) / P S L(2, \mathbb{R})$. Hitchin [2] has studied the spaces where $\operatorname{PSL}(2, \mathbb{R})$ is replaced by an arbitrary semi-simple group $G$. We will be interested in the case when $G=P S L(n, \mathbb{R})$. This space has many disconnected components which includes a certain component (called the Teichmuller component) which has dimension $(2 g-2) \operatorname{dim}(G)$. For $G=P S L(n, \mathbb{R})$, we denote the Teichmuller component by $\mathcal{T}_{n}(\Sigma)$. We would first like to observe that the dimension of the Teichmuller space of $W A_{n-1}$-strings is the same as that of $\mathcal{T}_{n}(\Sigma)$. Second, for the case $n=2$, this reduces to the usual Teichmuller space as we have already observed. We propose that the Teichmuller spaces $\mathcal{T}_{n}(\Sigma)$ introduced by Hitchin are the Teichmuller spaces for $W$-gravity.

Hitchin obtains the Teichmuller spaces as the space of solutions of the self-duality equations. These equations can be obtained from four dimensional self-dual YangMills by dimensional reduction[3]. The self-duality equations are

$$
F_{A}+\left[\Phi, \Phi^{\dagger}\right]=0
$$

where $A$ is a unitary connection on a holomorphic vector bundle $V, F_{A}$ its field strength and $\Phi$ is a holomorphic section of End $V \otimes K . \Phi$ is called a Higgs field and $K$ is the canonical line bundle. The holomorphicity condition on $\Phi$ is

$$
d_{A}^{\prime \prime} \Phi=0
$$

It is important to note that the self-duality equations are conformally invariant, i.e., they only depend on the conformal class of the metric. The different Teichmuller spaces obtained by Hitchin correspond to various choices of the Higgs pair $(V, \Phi)$. For example, in the $P S L(2)$ case, $V=\left(K^{-\frac{1}{2}} \oplus K^{\frac{1}{2}}\right)$ and $\Phi=\left(\begin{array}{cc}0 & h d z \\ 0 & 0\end{array}\right)$. In this case, the self-duality equations (11) can be shown to be equivalent to the constant negative curvature condition[包].

A first attempt to relate Higgs bundles to W-gravity was made by de Boer and Goeree (dBG) 国. dBG pointed out that the Higgs bundle setting was the way to 
generalise their "covariant" constructions for pure $W_{n}$-gravity to higher genus Riemann surfaces. However, their construction is somewhat indirect and involves the introduction of a somewhat arbitrary "reference connection".

In this letter, we attempt to establish that the spaces $\mathcal{T}_{n}(\Sigma)$ are indeed the Teichmuller spaces of $W_{n}$ gravity. We begin directly in the Higgs bundle setting, by identifying components of the Higgs field with a generalisation of the vielbein and the $S O(n)$ connection with a generalisation of the spin - connection. Conceptually this is an important point since it provides a way of understanding various formulations of $W_{n}$-gravity since the vielbeins and spin-connections give a covariant description of $W_{n}$-gravity. In principle, this gives a means of obtaining the equivalent of "general" coordinate transformations for W-gravity. We can also obtain a direct relationship to the "metric" formulations of W-gravity a la Hull and others [5]. This is in a sense a dual picture of the construction of $\mathrm{W}$-generators as higher order Casimirs [6]. Projective connections are obtained as geometric constructs without any direct reference to matter. This is essential for a geometric picture. Finally, we suggest that just as the uniformisation of Riemann surfaces is related to flat $\operatorname{PSL}(2, \mathbb{R})$ connections, there must exist such a picture for the $W_{n}$-case.

In another paper [7], Aldrovandi and Falqui have discussed the relation of Higgs bundles to Toda systems and hence through that to formulations of $\mathrm{W}$-gravity in a "liouville"-like formulation. They have also recognised the relationship of Higgs bundles to an uniformisation problem. However we believe that in our formulation of the problem there is a more explicit geometric structure that is revealed, that is also more general.

The paper is organised as follows. In section 2, we use the bosonic string to illustrate how the identification of vielbein with the Higgs field and the connection $A$ with the spin connection works. In section 3 , we discuss the $W_{n}$ case using the $W_{3}$ as an explicit example. In section 4, we relate to the works of [8, 9, 10, 团 by explicitly obtaining $W$-diffeomorphisms in our formulation. Finally, in section 5, we conclude 
with a brief discussion on these results and provide some suggestions.

\section{The bosonic string}

In this section, we shall demonstrate how our identifications of the vielbein as well as the spin-connection works in the case of the bosonic string. In addition, this will also illustrate self-duality equations. Consider, the case where the vector bundle $V=K^{-\frac{1}{2}} \oplus K^{\frac{1}{2}}$ and

$$
\Phi=\left(\begin{array}{cc}
0 & h d z \\
0 & 0
\end{array}\right)
$$

Note that $\operatorname{dim}(V)=2$ which implies that the connection $A$ in the self-duality equations is a $S U(2)$ connection'. Substituting for $\Phi$ in the self-duality equations gives

$$
F_{A}=\left(\begin{array}{cc}
\frac{1}{2} F^{0} & 0 \\
0 & -\frac{1}{2} F^{0}
\end{array}\right)=\left(\begin{array}{cc}
-1 & 0 \\
0 & 1
\end{array}\right) h^{2} d z \wedge d \bar{z}
$$

One can easily see from (4) that since $F^{ \pm}=0$. This implies that the $S U(2)$ connection is reducible to a $U(1)$ connection. This $U(1)$ connection can be identified with the spin-connection. It then follows that $F^{0}$ corresponds to the curvature and hence (4) is the constant negative curvature condition.

The connection $\mathcal{A}$ defined by

$$
\mathcal{A} \equiv\left(A+\Phi+\Phi^{\dagger}\right)
$$

is a flat $S L(2, \mathbb{C})$ connection. The flatness condition follows from the self-duality equation (11) and the condition that $\Phi$ is holomorphic (Eqn. (2)). Corlette and Donaldson have proved the converse of the above statement 11, 12. They have shown that if $V$ is a vector bundle on a Riemann surface $\Sigma$ with a completely reducible flat connection $\mathcal{A}$, then there exists a section (gauge), where $\mathcal{A}=\left(A+\Phi+\Phi^{\dagger}\right)$ where $(A, \Phi)$ satisfy the self-duality equations.

\footnotetext{
${ }^{1}$ The existence of such a connection requires that a certain stability condition has to be satisfied 3 . For all the situations we discuss in this paper, we have a stable Higgs bundle[2].
} 
Given a flat connection with gauge group $G$, it can be used to construct a map from $\pi_{1}(\Sigma)$ to the group $G$ by using the Wilson loop operator. The flatness of the connection ensures that the map depends only on the homotopy class of the path. See [13] for a discussion. Since the Wilson loop is invariant under conjugation, we obtain an element of $\operatorname{Hom}\left(\pi_{1}(\Sigma) \rightarrow G\right) / G$.

Returning to our example, Hitchin has shown that for this choice of Higgs field, the holonomy data is contained in real form (isomorphic to $\operatorname{PSL}(2, \mathbb{R})$ ) of $\operatorname{PSL}(2, \mathbb{C})$. Hence, for purposes of the holonomy data, we can represent the connection $\mathcal{A}$ by a flat $\operatorname{PSL}(2, \mathbb{R})$ connection. In summary, the Higgs bundle is associated to a flat $P S L(2, \mathbb{R})$ connection.

Now modify the Higgs field to

$$
\Phi=\left(\begin{array}{cc}
0 & h \\
\frac{a}{h} & 0
\end{array}\right),
$$

where $a \in \operatorname{Hom}\left(K^{-\frac{1}{2}}, K^{\frac{1}{2}}\right) \otimes K \sim K^{2}$. Hence, $a$ is a holomorphic quadratic differential. Again, the self-duality equations give the constant negative curvature condition[3]. We know that the space of constant negative curvature metrics is the Teichmuller space. We have also shown that the space of self-duality equations with $\Phi=\left(\begin{array}{cc}0 & h \\ \frac{a}{h} & 0\end{array}\right)$ gives the space of constant curvature metrics on $\Sigma$ and hence is gives Teichmuller space.

We have already identified the $U(1)$ connection with the spin-connection. We now identify the Higgs field with the vielbein of the Riemann surface. This identification was first made in [13]. The $\operatorname{PSL}(2, \mathbb{R})$ connection can then be explicitly written in terms of the vielbein and spin-connection as

$$
\mathcal{A}=\left(\begin{array}{cc}
\frac{\omega}{2} & \frac{e^{+}}{\sqrt{2}} \\
\frac{e^{-}}{\sqrt{2}} & \frac{-\omega}{2}
\end{array}\right),
$$

where $\omega$ is the spin-connection and $e^{ \pm}$are the vielbein. The flatness condition on the vielbein are nothing but the usual torsion constraints imposed on them. Comparing with the explicit form of $\Phi$ in eqn. (3), we see that the flatness condition reduces to 
the self-duality equations in the "conformal gauge" where $e_{z}^{+}=e_{\bar{z}}^{-}=h, e_{z}^{-}=e_{\bar{z}}^{+}=0$. $(+,-)$ are the tangent space indices and $(z, \bar{z})$ are Einstein indices. The fact that the vielbein and the spin-connection can be combined into a $P S L(2, \mathbb{R})$ gauge field (provided the constant negative curvature condition as well as the torsion constraints are imposed) has been observed in [14, 15]. The $P S L(2, \mathbb{R})$ transformations given below

$$
\begin{aligned}
& \delta e_{\mu}^{+}=\partial_{\mu} \epsilon^{+}-\omega_{\mu} \epsilon^{+}+\alpha e_{\mu}^{+}, \\
& \delta e_{\mu}^{-}=\partial_{\mu} \epsilon^{-}+\omega_{\mu} \epsilon^{-}-\alpha e_{\mu}^{-}, \\
& \delta \omega_{\mu}=\partial_{\mu} \alpha-e_{\mu}^{+} \epsilon^{-}+e_{\mu}^{-} \epsilon^{+},
\end{aligned}
$$

then correspond to diffeomorphisms (with parameters $\xi^{\mu}$ ) provided we choose

$$
\epsilon^{a}=\xi^{\mu} e_{\mu}^{a} \quad, \quad \alpha=\xi^{\mu} \omega_{\mu}
$$

The flatness conditions are now given below. As can be clearly seen, the first expression are the usual torsion free condition on the vielbein.

$$
\begin{aligned}
& T^{ \pm} \equiv d e^{+} \mp \omega \wedge e^{+}=0, \\
& F^{0} \equiv d \omega=-e^{+} \wedge e^{-} .
\end{aligned}
$$

In the vielbein formulation, the metric is given by

$$
g_{\mu \nu}=e_{\mu}^{a} \delta_{a b} e_{\nu}^{b} \sim \operatorname{tr}\left(E^{2}\right)
$$

where $E \equiv \frac{1}{\sqrt{2}}\left(\begin{array}{cc}0 & e^{+} \\ e^{-} & 0\end{array}\right)=\Phi+\Phi^{\dagger}$. This object is a $U(1)$ invariant symmetric two-tensor.

The Polyakov path integral for pure gravity can be written in terms of vielbeins as follows

$$
\int \frac{[\text { space of invertible vielbeins }]}{[\text { diffeomorphisms }] \times[\text { Lorentz }]} \quad e^{-\mu \int e^{+} \wedge e^{-}},
$$

where $\mu$ is the cosmological constant. 


\section{The $W$-string}

In this section, we will discuss the general case using $W_{3}$ to provide more details. However, we shall try to preserve generality as much as possible. Following Hitchin[2], we consider the vector bundle $V$ given by

$$
V=S^{n-1}\left(K^{-\frac{1}{2}} \oplus K^{\frac{1}{2}}\right)=K^{-\frac{n-1}{2}} \oplus K^{-\frac{n-3}{2}} \oplus \cdots \oplus K^{\frac{n-1}{2}}
$$

and choose the Higgs field

$$
\Phi=\left(\begin{array}{ccccc}
0 & h & 0 & \ldots & 0 \\
\frac{a_{1}}{h} & \ddots & \ddots & \ddots & \vdots \\
\frac{a_{2}}{h^{2}} & \ddots & \ddots & h & 0 \\
\vdots & \ddots & \frac{a_{1}}{h} & 0 & h \\
\frac{a_{n-1}}{h^{(n-1)}} & \cdots & \frac{a_{2}}{h^{2}} & \frac{a_{1}}{h} & 0
\end{array}\right) d z
$$

where $a_{i} \in K^{i+1}$. $(V, \Phi)$ form a stable Higgs pair. Again $\mathcal{A} \equiv A+\Phi+\Phi^{\dagger}$ is a flat $P S L(n, \mathbb{C})$ connection provided the self-duality equations are satisfied. However, the flat connection $\mathcal{A}$ has its holonomy contained in a split real form of $P S L(n, \mathbb{C})$ which is isomorphic to $\operatorname{PSL}(n, \mathbb{R})$.

Due to a theorem of Hitchin[2], one of the connected components of the space $\operatorname{Hom}\left(\pi_{1}(\Sigma) ; P S L(n, \mathbb{R})\right) / P S L(n, \mathbb{R})$ (which is the same as the space of solutions of the self-duality equations for the Higgs pair $(V, \Phi)$ just described) has dimension $(2 g-2) \operatorname{dim} P S L(n, \mathbb{R})=\left(n^{2}-1\right)(2 g-2)$ which is what one expects as the dimension of the Teichmuller space of $W A_{n-1}$ gravity. Further, the Higgs field is parametrised by $a_{i}$ which correspond to holomorphic quadratic, cubic, quartic, ..., differentials 2 . We would like to identify this space with the Teichmuller space of $W A_{n-1}$ gravity.

Following the bosonic string example in the previous section, we shall now identify the Higgs field with generalised vielbein and the gauge field $A$ with a generalised

\footnotetext{
${ }^{2}$ The zero modes of the $b$ anti-ghosts in the conformal gauge are in one to one correspondence to quadratic and higher differentials.
} 
spin-connection. For example, in the $W A_{2}$ string, the spin-connection is a $S O(3)$ connection and the vielbein have five components. The $P S L(3, \mathbb{R})$ connection can be parametrised as follows

$$
\mathcal{A}^{T}=\left(\begin{array}{ccc}
\omega-\frac{e^{+-}}{\sqrt{3}} & \left(\omega^{-}+e^{-}\right) & \sqrt{2} e^{--} \\
\left(\omega^{+}-e^{+}\right) & \frac{2 e^{+-}}{\sqrt{3}} & \left(-\omega^{-}+e^{-}\right) \\
\sqrt{2} e^{++} & \left(-\omega^{+}-e^{+}\right) & -\omega-\frac{e^{+-}}{\sqrt{3}}
\end{array}\right)
$$

where $\omega, \omega^{ \pm}$form the $S O(3)$ connection and $e^{A}$ are the generalised vielbein. We would like to make the following observations. We shall provide details in a later publication [19].

(i) Here, $\left(e^{ \pm}, \omega\right)$ form a $P S L(2, \mathbb{R})$ connection which is embedded in $P S L(3, \mathbb{R})$.

(ii) $e^{++}, e^{--}, e^{+-}$are the new vielbein. They have been labelled by their $U(1)$ charges (w.r.t. $\omega)$. Schoutens et al. introduced W-vielbeins which appear to correspond to $e^{++}$and $e^{--}$but not for $e^{+-}$in their covariant construction of an action for scalar fields coupled to $W A_{2}$ gravity 16.

(iii) Relaxing the curvature zero condition on the $S O(3)$ spin-connection, we obtain a more general setting for $W_{3}$-geometry. Just as the full general coordinate and Weyl transformations could be recovered by relaxing the constant curvature condition, we can recover the same in this case. W-Weyl transformations can be obtained by suitably generalising a procedure due to Howe[17].

(iv) In the $W A_{2}$ case, one can construct the "metric" $g_{2}$ from the quadratic $S O(3)$ invariant and a "cubic tensor" $g_{3}$ from the cubic $S O(3)$ invariant. This is done by introducing $E$ which consists of only the vielbein terms in the $\operatorname{PSL}(3, \mathbb{R})$ connection. Then

$$
g_{2} \sim \operatorname{tr}\left(E^{2}\right) \quad, \quad g_{3} \sim \operatorname{tr}\left(E^{3}\right)
$$

(v) The geometry is not Riemannian anymore in the sense that the torsion constraints are not sufficient to determine the connection in terms of the vielbein. 
For e.g., in the $W A_{2}$ case, there are 5 torsion constraints and the connection has 6 components. However, we can always choose a gauge where we trade one of the gauge symmetries to determine the connection in terms of the vielbein.

(vi) Conformal gauge corresponds to choosing

$$
e_{z}^{+}=e_{\bar{z}}^{-}=h \quad, \quad e_{z}^{+-}=e_{\bar{z}}^{+-}=0,
$$

which fixes the $S O(3)$ gauge freedom. Further, these gauge choices are algebraic and hence their corresponding ghosts can be ignored (since they would be noninteracting). Next, make the following gauge choices

$$
e_{\bar{z}}^{+}=e_{z}^{-}=e_{\bar{z}}^{++}=e_{z}^{--}=0
$$

whose corresponding ghosts (anti-ghosts) are of spin $-1,-2(2,3)$ as required for $W A_{2}$ gravity. Further, the residual transformations which preserve this gauge choice correspond to holomorphic (anti - holomorphic) transformations $\epsilon^{+}, \epsilon^{++}\left(\epsilon^{-}, \epsilon^{--}\right)$. The residual gravity degrees are freedom are $h, e_{z}^{++} \equiv v^{+}$and $e_{\bar{z}}^{--} \equiv v^{-}$

\section{$4 \quad W$-diffeomorphisms}

In this section, we shall identify $\operatorname{PSL}(n, \mathbb{R})$ gauge transformations with $W$ - diffeomorphisms provided a certain constraint is satisfied. Further, in contrast to earlier approaches, we obtain $W$-diffeomorphisms in a purely geometric fashion without invoking the presence of matter fields. In a different manner, the work of Gerasimov et al. [8] and subsequently that of Bilal et al. [9] discussed $W$-diffeomorphisms in the conformal gauge, as deformations of certain flag manifolds associated with jet bundles. In their construction, the action of $W A_{(n-1)}$ diffeomorphisms on the vector space $V=S^{n-1}\left(K^{-\frac{1}{2}} \oplus K^{\frac{1}{2}}\right)$ was demonstrated.

\footnotetext{
${ }^{3}$ These ideas have been further developed subsequently by Zucchini 18$]$.
} 


\subsection{The $P S L(2, \mathbb{R})$ case}

As usual, the bosonic $(P S L(2, \mathbb{R}))$ case shows us the way. Choose the Higgs field $\Phi$ as in eqn. (5). Consider the fields $\left(\tilde{\psi}_{1}, \tilde{\psi}_{2}\right) \in\left(K^{-\frac{1}{2}}, K^{\frac{1}{2}}\right)$ subject to the conditions

$$
\begin{aligned}
& \left(d_{\omega}^{\prime \prime}+\Phi^{\dagger}\right)\left(\begin{array}{c}
\tilde{\psi}_{1} \\
\tilde{\psi}_{2}
\end{array}\right)=0 \\
& \left(d_{\omega}^{\prime}+\Phi\right)\left(\begin{array}{c}
\tilde{\psi}_{1} \\
\tilde{\psi}_{2}
\end{array}\right)=0
\end{aligned}
$$

Eqn. (14) implies that the fields $\psi_{i}$ are holomorphic while the second eqn. (15) is a constraint on $\psi_{i}$. Interestingly, the self-duality equations (11) and (2) are implied by the consistency of the two conditions we have just imposed. Hence, self-duality equations correspond to the integrability of equations (14) and (15). The holomorphicity condition (2) on $\Phi$ implies that

$$
\omega_{z}=-h^{-1} \partial_{z} h \quad, \quad \omega_{\bar{z}}=h^{-1} \partial_{\bar{z}} h
$$

and that $a$ is holomorphic $\left(\partial_{\bar{z}} a=0\right)$. We shall now rescale the fields $\psi_{i}$ as follows

$$
\left(\begin{array}{c}
\tilde{\psi}_{1} \\
\tilde{\psi}_{2}
\end{array}\right)=\left(\begin{array}{c}
h^{\frac{1}{2}} \psi_{1} \\
h^{-\frac{1}{2}} \psi_{2}
\end{array}\right) \text {. }
$$

$\left(\psi_{1}, \psi_{2}\right)$ correspond to primary fields in the CFT sense, i.e., they have Einstein indices. This rescaling now enables us to make contact with diffeomorphisms in CFT. Further, the Christoffel connection are given by $\Gamma_{z z} z=2 \omega_{z}$ and $\Gamma_{\bar{z} \bar{z}} \bar{z}=2 \omega_{\bar{z}}$. A simple calculation shows that (14) and (15) translate to the following conditions

$$
\begin{aligned}
\left(\partial_{\bar{z}}+\mu \partial_{z}-\frac{1}{2} \partial_{z} \mu\right) \psi_{1} & =0 \\
\left(\partial_{z}^{2}-u\right) \psi_{1} & =0
\end{aligned}
$$

where $\mu \equiv \frac{\bar{a}}{h^{2}}$ is the Beltrami differential and $u \equiv \frac{1}{2} \partial_{z} \Gamma_{z z} z+\frac{1}{4}\left(\Gamma_{z z} z\right)^{2}-a$. It is a simple exercise to check that $u$ transforms like the Schwarzian. A similar observation was made by Sonoda 20] who pointed out that such a term could be added to the energy 
momentum tensor to make it transform like a $(2,0)$ tensor. See also, 21]. Hence, $u$ behaves like a projective connection. Eqn. (18) implies that $\psi_{1}$ transforms as

$$
\delta \psi_{1}=\xi^{z} \partial_{z} \psi_{1}-\frac{1}{2}\left(\partial_{z} \xi^{z}\right) \psi_{1}
$$

which is the standard transformation of a $\left(-\frac{1}{2}, 0\right)$ tensor in CFT. This is somewhat similar to what has been done in [8, 9]. However, there are some differences. The flatness condition that has been considered in [8, 9] is different from the flatness condition implied by the self-duality equations. The $P S L(2, \mathbb{R})$ gauge field is a completely geometric object with no relation apriori to matter fields. However, a special combination of the spin-connection transforms like the Schwarzian. This combination can be related to the stress-tensor via Ward identities considered by Verlinde[15, 8, 9]. It can also be seen that $W$-transformations have a presentation here without directly involving "matter fields."

The compatibility of conditions (18) and (19) implies that

$$
\left[\partial_{\bar{z}}+\mu \partial_{z}+2\left(\partial_{z} \mu\right)\right] u=\frac{1}{2} \partial_{z}^{3} \mu
$$

which is equivalent to the standard OPE for the stress-tensor (following a procedure outlined in [8].)

$$
T(z) T(w) \sim \frac{c / 2}{(z-w)^{4}}+\frac{2 T(w)}{(z-w)^{2}}+\frac{\partial_{w} T(w)}{(z-w)}+\ldots,
$$

provided we identify

$$
u \longrightarrow \frac{6}{c}\langle T\rangle
$$

Thus we have recovered the residual diffeomorphism in the conformal gauge. 


\subsection{The $W_{3}$ case}

We shall now repeat the exercise of the previous section for the $W A_{2}$ case. Consider the multiplet $\left(\tilde{\psi}_{1}, \tilde{\psi}_{2}, \tilde{\psi}_{3}\right) \in\left(K^{-1}, K^{0}, K\right)$. The Higgs field is given by (12) to be

$$
\Phi=\left(\begin{array}{ccc}
0 & h & 0 \\
\frac{a}{h} & 0 & h \\
\frac{b}{h^{2}} & \frac{a}{h} & 0
\end{array}\right) d z+\mathcal{O}\left(a^{2}, a b, b^{2}\right)
$$

This corresponds to setting $v^{ \pm}=0$ in the notation of section 3 . We shall not turn them on for simplicity. The holomorphicity condition (2) implies that

$$
\omega_{\mu}^{+}=\omega_{\mu}^{-}=0 \quad, \quad \omega_{z}=-h^{-1} \partial_{z} h \quad, \quad \omega_{z}=h^{-1} \partial_{\bar{z}} h
$$

and that $(a, b)$ are holomorphic $\left(\partial_{\bar{z}} a=\partial_{\bar{z}} b=0\right)$. The above solution is valid to $\mathcal{O}\left(a^{2}, a b, b^{2}\right)$. Impose the following conditions on $\left(\tilde{\psi}_{1}, \tilde{\psi}_{2}, \tilde{\psi}_{3}\right)$.

$$
\begin{gathered}
\left(d_{\omega}^{\prime \prime}+\Phi^{\dagger}\right)\left(\begin{array}{c}
\tilde{\psi}_{1} \\
\tilde{\psi}_{2} \\
\tilde{\psi}^{3}
\end{array}\right)=0 \\
\left(d_{\omega}^{\prime}+\Phi\right)\left(\begin{array}{c}
\tilde{\psi}_{1} \\
\tilde{\psi}_{2} \\
\tilde{\psi}_{3}
\end{array}\right)=0
\end{gathered}
$$

Eqn. (23) implies that the fields $\tilde{\psi}_{i}$ are holomorphic while the second eqn. (24) is a constraint on $\tilde{\psi}_{i}$. We shall now rescale the fields $\tilde{\psi}_{i}$ as follows

$$
\left(\begin{array}{c}
\tilde{\psi}_{1} \\
\tilde{\psi}_{2} \\
\tilde{\psi}^{3}
\end{array}\right)=\left(\begin{array}{c}
h \psi_{1} \\
\psi_{2} \\
h^{-1} \psi^{3}
\end{array}\right) \text {. }
$$

$\left(\psi_{1}, \psi_{2}, \psi_{3}\right)$ are the primary fields of CFT. Conditions (23) and (24) translate to the following conditions

$$
\begin{gathered}
\left(\partial_{\bar{z}}-\mu \partial_{z}+\partial_{z} \mu+\rho\left(\partial_{z}^{2}-\frac{2}{3} \tilde{u}_{2}\right)-\frac{1}{2} \partial_{z} \rho \partial_{z}+\frac{1}{6}\left(\partial_{z}^{2} \rho\right)\right) \psi_{1}=0 \\
\left(\partial_{z}^{3}-\tilde{u}_{2} \partial_{z}-\left(u_{3}+\frac{1}{2} \partial_{z} \tilde{u}_{2}\right)\right) \psi_{1}=0
\end{gathered}
$$


where $\mu \equiv \frac{a}{h^{2}}$ and $\rho \equiv \frac{b}{h^{4}}$ are the Beltrami differentials. Further,

$$
\tilde{u}_{2}=2 \partial_{z} \Gamma_{z z}^{z}+\left(\Gamma_{z z}^{z}\right)^{2}+2 a \quad, \quad u_{3}=\left[\partial_{z}-2 \Gamma_{z z}^{z}\right]\left(\omega_{z}^{-} h\right)-b \quad,
$$

where we have restored dependence on $\omega_{z}^{-}$(even though it is vanishing to $\mathcal{O}\left(a^{2}, a b, b^{2}\right)$ ). This is to show that combinations of the connection behave like a generalised projective connection in the sense of [8, 9].

Equations (26) and (27) are identical to those obtained in [8, see eqn. (35)]. As discussed earlier, we can continue to use arguments identical to theirs and show that the these two equations are equivalent to the following OPE's.

$$
\begin{aligned}
T(z) T(w)= & \frac{c / 2}{(z-w)^{4}}+\frac{2 T(w)}{(z-w)^{2}}+\frac{\partial_{w} T(w)}{(z-w)}+\ldots \\
T(z) W(w)= & \frac{3 W(w)}{(z-w)^{2}}+\frac{\partial_{w} W(w)}{(z-w)}+\ldots \\
W(z) W(w)= & \frac{c / 3}{(z-w)^{6}}+\left(\frac{2}{(z-w)^{4}}+\frac{\partial_{w}}{(z-w)^{3}}+\frac{3}{10} \frac{\partial_{w}^{2}}{(z-w)^{2}}\right. \\
& \left.+\frac{1}{15} \frac{\partial_{w}^{3}}{(z-w)}\right) T(w)+\frac{16}{5 c}\left(\frac{2}{(z-w)^{2}}+\frac{\partial_{w}}{(z-w)}\right) \Lambda(w)
\end{aligned}
$$

where $\Lambda=T^{2}$. The above OPE corresponds to the semi-classical limit $(c \rightarrow \infty)$ of the OPE's of the $W_{3}$-algebra given in [1] after we make the following identifications

$$
\tilde{u}_{2} \longrightarrow \frac{24}{c}\langle T\rangle \quad, \quad u_{3} \longrightarrow \frac{24}{c}\langle W\rangle
$$

Equation (26) implies the following transformation law for a $(-1)$ - differential

$$
\delta \psi_{1}=\xi^{z} \partial_{z} \psi_{1}-\left(\partial_{z} \xi^{z}\right) \psi_{1}+\xi^{z z}\left(\partial_{z}^{2} \psi_{1}\right)-\frac{1}{2}\left(\partial_{z} \xi^{z z}\right)\left(\partial_{z} \psi_{1}\right)+\left(\left(\frac{1}{6} \partial_{z}^{2}-\frac{2}{3} \tilde{u}_{2}\right) \xi^{z z}\right) \psi_{1}
$$

where $\xi^{z}$ and $\xi^{z z}$ (that parametrise $W$-diffeomorphisms) are given by $\xi^{z}=\epsilon^{+} h^{-1}$ and $\xi^{z z}=\epsilon^{++} h^{-2} \cdot \epsilon^{+}$and $\epsilon^{++}$are $S L(3, \mathbb{R})$ gauge parameters.

We have demonstrated how $W$-diffeomorphisms are obtained in our formulation. The interesting feature is that certain combinations of the generalised connection play the role of projective connections. This is not surprising since these connections are required to form covariant derivatives under transformations which are much larger than the set of projective transformations. 


\section{Conclusion and Outlook}

In this letter, we have demonstrated that the Teichmuller spaces $\mathcal{T}_{n}(\Sigma)$ introduced by Hitchin as the space of solutions of the self-duality equations are indeed the Teichmuller spaces for $W$-gravity. Even though we have restricted ourselves to the case of $W A_{n}$-gravity in this paper, this generalises to $W$-gravity related to other semi-simple groups. By introducing generalised vielbeins and connections, we are now able to give a gauge independent descriptions of $W$-gravity. For the case of $W$-string theory, one can now write a Polyakov path integral. This is done by considering the path integral over the space of all generalised vielbein with the gauge symmetry corresponding to generalised diffeomorphisms and generalised local Lorentz transformations. The conformal and light-cone gauges can be obtained by appropriately gauge fixing these symmetries. In order to relate to the "metric" formulation [5, 23], we have suggested that the symmetric tensors are given by higher order invariants.

$$
g_{n} \sim \operatorname{tr}\left(E^{n}\right)
$$

We have explicitly shown how $W$-diffeomorphisms as discussed by [8, 9] are reproduced in our formulation in the "conformal gauge." The interesting point is that special combinations of the generalised connections played the role of projective connections.

We have seen that the generalised vielbeins and connections in W-string theory can be represented by flat $P S L(n, \mathbb{R})$ connections for the case of surfaces with genus $g>1$. What are the groups to be chosen for the sphere and torus topology? We shall use the bosonic case as well as the dimension of the group as a guide to figure out the answer. In the bosonic case, the group is $S O(3) \sim S U(2) / Z_{2}$ for spherical topology and $I S O(2) \sim I U(1)$ for torus topology . This leads us to guess that the groups $S U(n)$ for odd ' $n$ ' and $S U(n) / Z_{2}$ for even ' $n$ ' for spherical topology and $I U(n-1)$ for

\footnotetext{
${ }^{4} I U(n)$ is the inhomogeneous group in $C^{n}$. It consists of all the elements of $U(n)$ together with the group of translations in $C^{n}$.
} 
torus topology. Both these groups have the right dimension $\left(=n^{2}-1\right)$. For example, $I S O(n)$ will not work in the torus case except for $n=2$. Further, the torus case can be obtained by contractions of either $S U(n)$ or $S L(n, \mathbb{R})$, just as in the bosonic case 22]. It is probably essential to solve the torus and sphere cases in detail for Wstrings in this language in order to understand the spectrum as well as the divergence structure of these theories.

To make further progress towards a description of W-strings the most important task seems to be understand the uniformisation associated with the flat $\operatorname{PSL}(3, \mathbb{R})$ connections. In the case of $\operatorname{PSL}(2, \mathbb{R})$ this naturally leads us to the upper halfplane with the Poincare metric and the Riemann surface is described by quotienting the upper half-plane by the Fuchsian group. String matter fields can be formulated as living on this space. The jet bundles of $K^{-\frac{1}{2}}$ are naturally associated with this uniformisation [3]. In the case of $\operatorname{PSL}(3, \mathbb{R})$ the natural structure is the one associated with the 2-jet bundle of $K^{-1}$. The uniformizing space in its most general form appears to be a space which is a domain in $C P^{2}$. We might expect therefore in the $\operatorname{PSL}(3, \mathbb{R})$ case a domain in $C P^{2}$ which when quotiented by the Fuchsian group (which is given by the embedding of the homotopy group of the surface into a discrete sub-group of $\operatorname{PSL}(3, \mathbb{R})$ ) describes the Riemann surface plus the additional data encoded in the new vielbeins and connections. This space would also naturally provide $w-$ coordinates. This structure can also straightforwardly extended to the general $W_{n}$ case'.

It is tempting however to try and relate the Hitchin construction for $\operatorname{PSL}(3, \mathbb{R})$ directly to Goldman's work on the convex real projective $\left(R P^{2}\right)$ structures on Riemann surfaces[25]. In recent work[26], Choi and Goldman have shown that the Teichmuller space of convex $R P^{2}$ structures is the same as the Teichmuller component

\footnotetext{
${ }^{5}$ Gervais et. al. have considered the possibility of surfaces embedded in $C P^{n}$ in the context of W-geometry and Toda systems 24, 18. Also, in a recent paper[7], the relationship of Toda systems to Higgs bundles was demonstrated.
} 
of the moduli space of Higgs bundles for $\operatorname{PSL}(3, \mathbb{R})$. Goldman also provides FenchelNielsen co-ordinates for his Teichmuller space by considering convex domains in $R P^{2}$ quotiented by a Fuchsian subgroup of $P S L(3, \mathbb{R})$. In contrast to the $P S L(2, \mathbb{R})$ case, there are two lengths (and corresponding twists) associated to every $P S L(3, \mathbb{R})$ nonconjugate element of the Fuchsian group. $(6 g-6)$ of these lengths ( and their twists) together with two other parameters associated with every pant in the corresponding pants decomposition provide the $(16 g-16)$ real co-ordinates of the Teichmuller space of convex $R P^{2}$ structures. The gluing prescription is also generalised to the $R P^{2}$ case, involving however only the lengths and twists. Some progress has also been made towards using these co-ordinates to write down a Weil-Petersson like measure on the Teichmuller spacer, which contains within it the usual Weil-Petersson measure on $\mathcal{T}_{2}$.

Even though the Higgs bundle approach appears to lead to an uniformisation by a domain in $C P^{2}$, the associated real holonomy should lead to a construction identical to that of Goldman. The challenge here is to formulate the matter part of W-string theory on this uniformizing space and provide the kind of explicit description available in standard string theory. In analogy with the bosonic string [27, 28], it would be interesting to study the structure of W-string theory at the boundaries of this Teichmuller space.

Acknowledgements: We would like to thank M. Blau, S. Das, S. F. Hassan, K. Joshi, P. Majumdar, D. S. Nagaraj, K.S. Narain, S. Nag, B. Rai, P. Sastry, A. Sen and S. Wadia for useful discussions. S. G. would like to thank the organisers of the International Colloquium on Modern Quantum Field Theory II at Tata Institute, Bombay (January 1994) and the Spring Workshop on Strings, Quantum gravity and Gauge theories at ICTP, Trieste (April 1994) for an opportunity to present some of these results. 


\section{References}

[1] A. B. Zamolodchikov, Teor. Mat. Fiz. 65 (1985) 1205.

V. A. Fateev and A. B. Zamolodchikov, Nuc. Phys. B280 (1987) 644.

[2] N. J. Hitchin, Topology 31 (1992) 451-487.

[3] N. J. Hitchin, Proc. London Math. Soc. 55 (1987) 59-126.

[4] J. de Boer and J. Goeree, Nuc. Phys. B401 (1993) 369.

[5] C. M. Hull, Commun. Math. Phys. 156 (1993) 245; Nuc. Phys.B 413 (1994) 296.

[6] F. A. Bais, P. Bouwknegt, K. Schoutens and M. Surridge, Nuc. Phys. B304 (1988) 348 .

[7] E.Aldrovandi and G.Falqui, "Geometry of Higgs and Toda Fields on Riemann Surfaces," Preprint hep-th/9312093.

[8] A. Gerasimov, A. Levin and A. Marshakov, Nuc. Phys. B360 (1991) 537-558.

[9] A. Bilal, V. Fock and I. Kogan, Nuc. Phys. B359 (1991) 635-672.

[10] de Boer and J. Goeree, Nuc. Phys. B381 (1992) 329-359; Phys. Lett. B274 (1992) 289-297.

K. Yoshida, Int. J. of Mod. Phys. A7 (1992) 4353-4376.

[11] K. Corlette, J. Diff. Geom. 28 (1988) 361-382.

[12] S. K. Donaldson, Proc. London Math. Soc. 55 (1987) 127-131.

[13] D. Birmingham, M. Blau, M. Rakowski and G. Thompson, Physics Reports 209 (1991) 129-340. 
[14] D. Montano and J. Sonnenschein, Nuc. Phys. B324 (1989) 348.

E. Verlinde and H. Verlinde, Nuc. Phys. B348 (1991) 435.

[15] H. Verlinde, Nuc. Phys. 337 (1990) 652.

[16] K. Schoutens, A. Sevrin and van Nieuwenhuizen, Phys. Lett. B243 (1990) 245249; Nuc. Phys. B349 (1991) 791-814.

[17] P. S. Howe, J. Phys. A12 (1979) 393.

[18] R. Zucchini, Class. Quant. Grav. 10 (1993) 253-278.

[19] S. Govindarajan and T. Jayaraman (in preparation).

[20] H. Sonoda, Nuc. Phys. B281 (1987) 546.

[21] T. Eguchi and H. Ooguri, Nuc. Phys. B282 (1987) 308-328.

[22] See chapter 10 in R. Gilmore, Lie Groups, Lie Algebras and some of their applications, John Wiley \& Sons, New York.

[23] For instance, see C. M. Hull, "Classical and Quantum W-gravity," in the proceedings of the summer school in High Energy Physics and Cosmology, 1992 (World Scientific) and references therein.

[24] J-L. Gervais and Y. Matsuo, Commun. Math. Phys. 152 (1993) 317-368.

[25] W. M. Goldman, J. Diff. Geom. 31 (1990) 791-845.

[26] S. Choi and W. M. Goldman, Proc. of the AMS 118 (1993) 657.

[27] E. D’Hoker and D. H. Phong, Rev. Mod. Phys. 60 (1988) 917.

[28] E. Gava, R. Iengo, T. Jayaraman and R. Ramachandran, Phys. Lett. B168 (1986) 207. 